\title{
Analysis of Research Trends Using G-power in Physiotherapy Research in Korea: Systematic Review
}

\author{
Ki Hyun Baek', Mi Soo Lim', Mi Ho Park', Hee Ae Seo', Dong A Hyeon'1, Hyoung-won Lim² \\ 'Department of Physical Therapy, Graduate School, Dankook University, Cheonan, Korea; ${ }^{2}$ Department of Physical Therapy, Dankook University, \\ Cheonan, Korea
}

Purpose: This study examined the research trends using G-power centered on major Korean registered sites and presents the research trends of studies with a high level of evidence.

Method: This study selected three research journals listed in the Korea Research Foundation among physical therapy journals in the field of physiotherapy. The selected papers were classified according to the following: 1) study type, 2) study level, and 3) quality of the study. Result: Sixty-nine articles were selected and classified according to the study type. The musculoskeletal system, central nervous system, and others comprised 73.91\% $(n=51), 17.39 \%(n=12)$, and 8.7\% $(n=6)$, respectively. The results were classified into 62 experimental studies, four observational analytic studies, and three descriptive studies. The experimental studies included 22 randomized controlled trials (level 2) and 40 non-randomized trials (level 3). Sixteen randomized controlled trials were classified for a qualitative evaluation, and 12 papers with a score of six or more (good) were found.

Conclusion: Many high-level studies were found in G-power, and the quality of the study was also good. Therefore, the effective number of samples obtained using G-power is one of the ways to enable high-quality research.

Keywords: G-power, Research trend, PEDro scale, Physiotherapy

\section{서 론}

로봇, 인공지능(AI), 나노기술(NT) 등 융합기술 발전으로 4 차 산업혁 명이 도래함에 따라, ${ }^{1}$ 최근 정부 및 관련 기관들을 중심으로 다양한 로봇 콘텐츠 기획 및 제작에 대한 지속적인 투자가 이루어지고 있다. ${ }^{2}$ 보건의료산업에서는 원거리 진료나 원격조정으로 정밀수술을 진행 하는 것과 더 나아가 인공지능이 환자를 진단할 수 있는 프로그램이 개발되고 있으며, 일부분은 시행하는 중이다. ${ }^{3}$ 이러한 눈부신 발전과 함께 물리치료분야 역시 발전을 거듭하고 있다. 뇌졸중 환자들의 치 료를 돕기 위해 팔꿈치 외골격 로봇을 설계하는 연구와 보행훈련을 도와주는 체중 지지 로봇을 이용한 보행 및 균형 능력 등에 관한 연 구들과 같이 물리치료분야에서 다양한 연구들이 시행되고 있다.,5

국가통계포털 KOSIS에 따르면 지난 2015년도 임상 물리치료사 31,194 명에서 2019년도 현재 임상 물리치료사는 41,457 명으로 증가하 였고, ${ }^{6}$ 최근 국내 물리치료 학술 등재지도 늘어나고 있다. 질환이 다 양해지고, 의료소비자의 인식이 높아지고, 환자 만족도의 기준이 높

Received Mar 13, 2020 Revised Apr 3, 2020

Accepted Apr 13, 2020

Corresponding author Hyoung-won Lim

E-mail movt12@hanmail.net
아짐에 따라 확립된 이론과 과학적인 기초를 바탕으로 치료사의 직 무능력은 향상되고 있다. 또한 빠르게 변화하는 의료현장에서 보다 쉽게 대응할 수 있도록 전공 심화과정도 습득하고 있는 실정이다. 근 거중심치료(evidence-based practice, EBP)라고 하는 환자 치료의 의사 결정에 있어 현존하는 최상의 근거에 관한 현명하고 명백한 적용을 통해 임상물리치료사와 물리치료 연구에 양적 성장과 더불어 질적 성장 또한 증가하고 있다.?

최근 국내 물리치료 연구에서는 연구가 어떤 목적을 갖고 있고, 그 연구에 누가 관심을 갖고 있으며, 이해관계가 있는지 등을 염두에 두 고 동료 연구자들과 연구의 진행과정에서 의견을 주고 받는 공동작 업과 과정을 거치면서, ${ }^{9}$ 이전보다 더 질 높은 연구와 정당성이 있는 연구 그리고 신뢰성이 높은 연구를 추구하고 있다. 특히 연구자들이 논문의 정당성을 갖추기 위해 연구를 계획 할 때 필요한 표본수(sample size)를 결정하는 것은 연구 결과의 정확도에 결정적인 역할을 한 다. ${ }^{8}$ 표본수 결정에 있어서 그 수가 충분하지 않았을 때 결과의 검정 력이 떨어져 의미있는 차이를 발견하기 어렵고 신뢰도가 떨어진다. 반 
대로 표본수를 크게 설정하였을 때에는 비용, 시간 등 제한된 자원의 낭비를 초래할 수 있으며, 연구대상자를 필요 이상의 위험에 노출시 킬 수 있다. 또한 연구자가 필요로 하는 변수뿐만 아니라 다른 변수까 지 통계적으로 유의하게 만들기 때문에 연구자가 연구에서 필요로 하는 변수가 무엇인지 알기 어려워 통계적으로 유의한 차이가 있을 수 있으나, 임상적인 유효함은 찾지 못할 수 있다.1012 일반적으로 통계 적인 유의한 차이가 나오지 않는 경우는 표본수가 부족하거나 실제 의 효과크기가 예상했던 효과크기보다 작은 경우이다.11,12 그러므로 얼마의 효과크기가 임상적으로 의미 있는가를 기준으로 연구하는 것이 연구의 질을 높일 수 있으며, 연구 계획에서 임상적으로 의미 있 는 최소한의 효과크기가 무엇인지 찾고, 그 기준으로 유의한 차이가 나타나는 표본수를 결정해야한다.11,12

보건의료분야의 실험연구 등 반복측정자료를 수집하는 연구를 진행할 때, 분석 방법에 따라 다양한 표본수 산출을 해야 할 것이다.13 계획된 연구에 대한 표본수 산정 및 검정력 계산을 위한 통계 프로그 램 중에는 소프트웨어의 프로그래밍에 대한 지식과 넓은 통계에 대 한 이해가 필요하고, 유료 상업적 프로그램을 사용해야 하는 경우가 많다.13 G-power는 이러한 지식을 요구하지 않는 graphic user interface (GUI) 기법을 사용해 실제 사용이 어렵지 않게 표본수 산출 및 검정 력을 계산할 수 있도록 하였다. ${ }^{14}$ 또한, G-power는 반복측정 자료에 대 한 분석의 표본수를 늘리지 않고, 검정력을 키울 수 있는데 이는 시 간의 흐름에 따른 변화 파악과 혼란변수의 영향을 감소시킬 수 있는 방법이기도 하다. ${ }^{14}$

우리가 아는 한 국내 물리치료 연구에서 G-power를 사용한 연구 는 제한적으로 시행되고 있으며, G-power를 사용한 연구동향 분석은 시도된 적이 없다. G-power를 사용하여 표본수를 산출한 연구는 적 절한 검정력을 제공할 수 있기 때문에 근거 수준이 높은 연구를 시행 할 수 있다. ${ }^{15}$ 따라서 G-power를 사용한 연구를 조사하여 연구의 질 적수준과 연구동향을 파악하는 것은 매우 필요하다. 본 연구의 목적 은 국내 물리치료 주요 등재지를 중심으로 G-power를 사용한 연구 동향을 분석하고 이를 통하여 근거수준이 높은 연구들의 연구동향 을 물리치료사와 연구자들에게 제시하고자 함이다.

\section{연구 방법}

\section{1. 자료수집}

본 연구는 국내 물리치료 연구에서 G-power를 사용한 연구동향 분 석을 위하여 학술 주제 분류에서 물리치료분야의 학회지 가운데 한 국연구재단 등재 학회지로 3 년 이상 된 학회지인 한국전문물리치료 학회지(Physical Therapy Korea, PTK), 대한물리치료학회지(Journal of Korean Physical Therapy, JKPT), 대한물리의학회지(Korean Society of
Physical Medicine, KSPM)를 대상으로 하였다. 출판된 논문의 원문은 각 저널의 홈페이지 http://www.ptkorea.org/index.php, http://www.kptjournal.org/main.html, http://www.jkspm.org/main.html에서 검색하여 수집하였다 (Figure 1).

\section{2. 분류방법}

본 연구에서 논문은 3 개의 국내 학회지에서 선정하였으며, 선정된 논 문은 1) 연구 형태, 2) 연구 수준,3) 연구의 질에 따라서 분류되었다. 연 구는 다시 근골격계 연구와 신경계 연구, 그리고 기타 연구로 분류하 였으며 진단을 받은 환자군과, 증상을 보인 일반인, 그리고 건강인으 로 분류하였다.

\section{1) 선정기준}

논문의 선정기준은 (1) 최근 5년 이내에 출판된 논문, (2) G-power를 사 용한 연구이다. 위의 기준에 따라 선정된 연구에서 연구의 질에 따른 분류를 위해 설정된 기준은 (1) 무작위 대조군 연구(Randomized Controlled Trial, RCT), (2) 증상을 보인 일반인을 대상으로 한 연구이다.

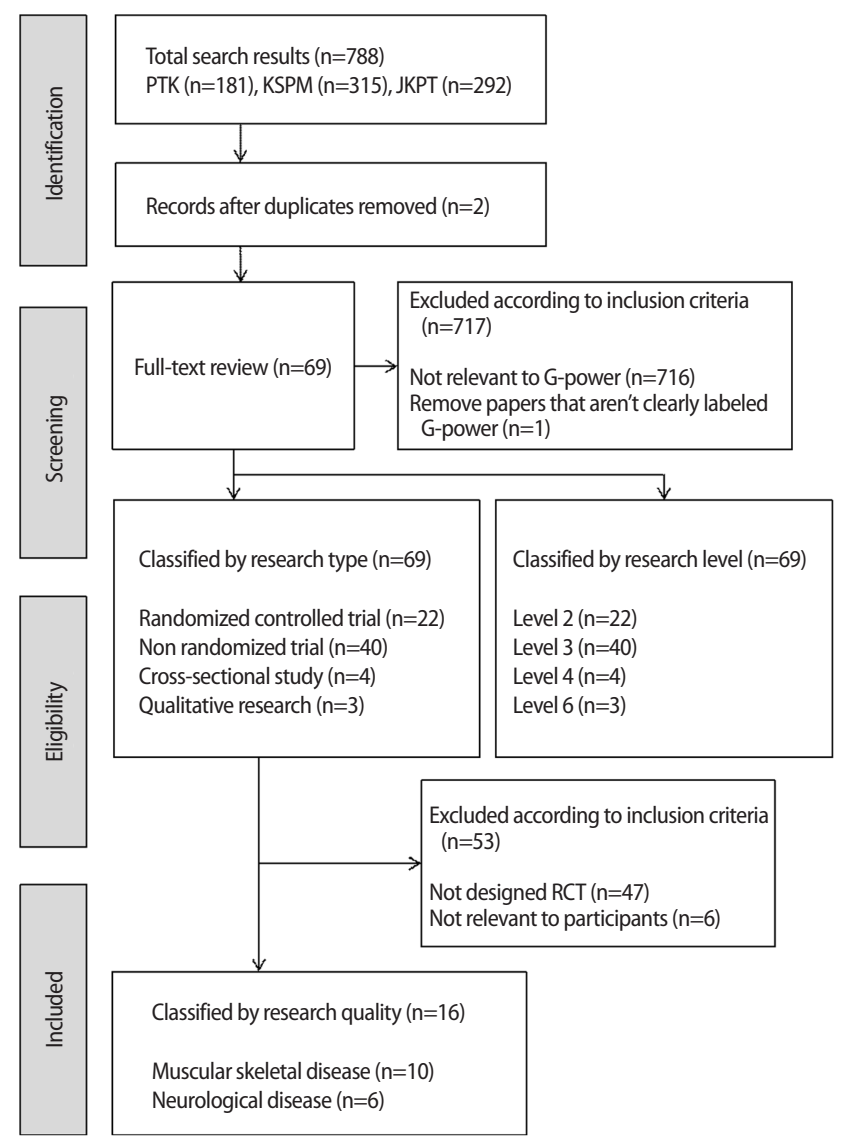

Figure 1. Flow diagram of systematic review. 


\section{2) 배제기준}

논문의 배제기준은 (1)기초연구, (2)동물실험 연구, (3)학회지 내 중복 된 논문, (4)G-power의 검정력이나 효과 크기등을 제시하지 않은 논 문이다. 연구의 질에 따른 분류를 위해 추가적으로 설정된 배제기준 은 (1)RCT연구 중 PEDro scale 1번 항목에 해당되지 않는 연구이다.

\section{3. 연구형태에 따른 분류}

연구형태에 따른 분류항목은 선행연구의 분류항목에 근거하여 설정 하였고, ${ }^{16}$ 연구의 목적에 맞게 변경하여 작성하였다. 본 연구에서는 기초연구와 동물실험 연구를 배제하여 최종 4가지 항목으로 분류되 었으며, 최종 설정한 분류항목은 고찰(review), 실험연구(experimental study), 관찰분석연구(observative analytic study), 기술적 연구(descriptive study)이다.

\section{4. 연구 수준}

선행연구에서 제시한 연구수준 분류는 총 8 단계로 구성되었지만, ${ }^{17,18}$ 본 연구에서는 기초연구와 동물실험 연구를 배제하여 총 6 단계로 구 성되었다. 이 분류는 근거기반연구의 수준을 나타내며, 1단계는 가장 높은 수준, 6 단계는 가장 낮은 수준으로 판단할 수 있다.

\section{5. 연구의 질적 평가}

연구의 질적 평가를 위해 RCT논문을 따로 분류하여 PEDro scale을 사용해 평가하였다. PEDro scale은 1번 항목을 충족해야 다음 항목으 로 넘어갈 수 있다. 1번 항목이 충족된 연구는 남은 항목에 항목별 유 무를 체크하였고, 조건이 충족되면 각 1점씩 부여하여 총 10 점 만점 으로 계산한다. 4점 미만 불량(poor), 4-5점 보통(fair), 6-8점 좋음 (good), 9-10점 매우 좋음(excellent)으로 평가하였다. 연구의 질적평가 는 두 명의 박사과정 연구자가 독립적으로 수행한 후 토의를 통해 결 론을 도출하였고, 의견 일치를 하지 못했을 경우 다른 연구자가 참여 해 결론을 도출하였다. ${ }^{19}$

\section{결 과}

\section{G-power를 사용한 연구 동향}

최근 5년 이내(2015-2019) 국내 주요 물리치료 학회지 3곳에서 출판 된 논문 중 G-power를 사용한 연구 동향을 분석하였을 때 결과는 다

\section{음과 같다(Table 1).}

\section{2. 연구형태에 따른 분류}

최근 5 년동안 게재 된 한국전문물리치료학회지 181 편, 대한물리치료 학회지 315편, 대한물리의학회지 292편 총 788편 중 G-power가 사용 된 논문은 한국전문물리치료학회지 20편, 대한물리치료학회지 33 편, 대한물리의학회지 16 편이었다. G-power가 사용된 72 편의 논문을 다시 선정기준에 의해 분류하였으며, 3편의 논문이 탈락되어 총 69편 의 논문이 선정되었다.

선정된 논문 69편을 근골격계, 신경계, 기타로 분류하였고 그 안에 서 대상자를 한번 더 분류하였다(Table 2). 분류된 논문 비율은 근골 격계 $73.91 \%$ (51편), 신경계 $17.39 \%$ (12편), 기타 $8.7 \%$ (6편)이었다. 근골격 계 논문에서는 진단 받은 환자를 대상으로 한 환자군(patient group) 논문, 진단을 받지 않은 일반인 중 증상을 보인자를 대상으로 한 증 상대조군(symptom group) 논문, 건강인을 대상으로 한 건강인 (healthy group) 논문으로 분류하였고, 환자군은 8편, 증상대조군 18 편, 건강인 25 편이었다. 신경계논문은 모두 환자군으로 12 편이었고, 기타논문에서 환자군은 1편, 건강인은 5 편이었다.

\section{3. 연구수준}

선정된 논문을 연구형태에 따라 분류한 결과 Experimental study 62 편, Observative Analytic study 4편, Descriptive study 3편으로 Experimental study가 가장 많았다. 실험 연구 62편은 randomized controlled trial 22편, non randomized trial 40편이었고, cross-sectional study 4편이 었으며, qualitative research 3편이었다(Table 3).

\section{4. 연구의 질적 평가}

G-power가 사용된 69편의 논문 중 연구의 질 평가를 위해 분류된 $\mathrm{RCT}$ 논문은 총 16 편으로 근골격계 환자를 대상으로 한 논문 10 편, 신

Table 1. Research trend using G-power

\begin{tabular}{lcccccc}
\hline $69 / 788$ & 2015 & 2016 & 2017 & 2018 & 2019 & Total \\
\hline PTK & $2 / 40$ & $4 / 38$ & $6 / 36$ & $3 / 29$ & $5 / 38$ & $20 / 181$ \\
KSPM & $0 / 54$ & $4 / 66$ & $6 / 62$ & $8 / 66$ & $15 / 67$ & $33 / 315$ \\
JKPT & $2 / 73$ & $4 / 64$ & $3 / 53$ & $4 / 42$ & $3 / 60$ & $16 / 292$ \\
Total & $4 / 167$ & $12 / 168$ & $15 / 151$ & $15 / 137$ & $23 / 165$ & $69 / 788$ \\
& $(2.40 \%)$ & $(7.14 \%)$ & $(9.93 \%)$ & $(10.95 \%)$ & $(13.94 \%)$ & $(8.76 \%)$ \\
\hline
\end{tabular}

Table 2. Classification by participant

\begin{tabular}{ccccc}
\hline & $\begin{array}{c}\text { Muscular skeletal disease } \\
(\mathrm{n}=51)\end{array}$ & $\begin{array}{c}\text { Neurological disease } \\
(\mathrm{n}=12)\end{array}$ & $\begin{array}{c}\text { The others disease } \\
(\mathrm{n}=6)\end{array}$ \\
\hline Patient group & Symptom group & Healthy group & Patient group & Patient group \\
$8(11.59 \%)$ & $18(26.08 \%)$ & $25(36.23 \%)$ & $12(17.39 \%)$ & $1(1.45 \%)$ \\
\hline
\end{tabular}


경계 환자를 대상으로 한 논문 6편이었다. 신경계 환자를 대상으로 한 6 편의 논문 중 4 편은 뇌졸중 환자를 대상으로 하였고, 1편은 파킨 슨 환자를 대상으로 한 연구로 대부분이 균형 및 보행에 미치는 영향 을 조사하였다. 나머지 1편은 소아를 대상으로 한 연구였으며 근 두 께와 대동작 기능을 보는 연구였다. 근골격계 논문 10 편은 목과 어깨 의 통증과 가동범위를 조사한 연구가 6 편이었고, 호흡재활 프로그램 이 가로막 움직임과 호흡기능에 미치는 영향을 보는 연구 1편, 허리의 통증과 골반의 움직임 및 균형을 보는 연구 1편, 무릎의 통증과 가동 범위 및 균형을 보는 연구 1 편, 발목의 불안정성과 가동범위 및 균형 을 보는 연구가 1편이었다. ${ }^{20-35}$

총 16 편의 논문을 PEDro scale 점수에 따라 분석한 결과, 10 점이 1편 (6.25\%), 9점이 1편(6.25\%), 8점이 1편(6.25\%), 7점이 1편(6.25\%), 6점이 8편

Table 3. Classification by research type and level

\begin{tabular}{llcc}
\hline Type of study (69) & \multicolumn{1}{c}{ Definition } & $\begin{array}{c}\text { Level of } \\
\text { study }\end{array}$ & $\mathrm{n}$ \\
\hline Review (0) & Systemic review & Level 1 & 0 \\
& Meta-analysis & Level 1 & 0 \\
Experimental study (62) & Randomized controlled trial & Level 2 & 22 \\
& Non randomized trial & Level 3 & 40 \\
& Cohort studied & Level 4 & 0 \\
Observative analytic study (4) & Case-controlled study & Level 5 & 0 \\
& Cross-sectional study & Level 5 & 4 \\
& Case reports & Level 6 & 0 \\
Descriptive study (3) & Narrative literature review & Level 6 & 0 \\
& Qualitative research & Level 6 & 3
\end{tabular}

(50\%), 5점이 4편(25\%)이었으며 평균 6.37점으로 나타났다. PEDro scale 의 질적 수준의 점수는 '좋음' 범주에 속하였다(Table 4).

\section{고 찰}

본 연구는 국내 주요 물리치료 학회지 3 곳에 출판된 논문 가운데 Gpower를 사용한 논문들의 연구 동향을 분석하기 위해 실시되었다. 총 788편의 출판 논문 중에서 G-power를 사용한 논문은 69편으로 $8.76 \%$ 에 해당하는 비교적 낮은 비율을 차지하는 것으로 나타났다. 그 러나 G-power 사용 추이는 2015년에는 2.4\%에서 2019년에는 13.94\% 로 $11.54 \%$ 가 증가하여 중요한 연구방법 수단으로 성장하였음을 알 수있다.

연구대상자에 관한 분류는 근골격계에서 건강인을 대상으로 연 구가 진행된 경우가 $36.23 \%$ (25편)로 가장 많았고, 두번째로 근골격계 에서 증상대조군을 대상으로 진행된 연구가 $26.08 \%$ (18편), 그 다음으 로는 신경계에서 환자군을 대상으로 한 연구가 $17.39 \%(12$ 편)로 많았 다. 특별히 근골격계분야에서 G-power를 사용한 연구가 많은 이유로 는 출판된 근골격계분야 논문의 대부분이 건강인을 대상으로 하여 대상자 모집이 신경계분야보다 수월했기 때문으로 사료된다. 그러나 대부분의 연구는 치료 효과와 관련이 있다고 할 수 있기 때문에, 대상 자의 확보가 어렵더라도 건강인을 대상으로 연구를 수행하는 것은 지양해야 할 것이다. 반면 신경계 연구에서 G-power 사용이 적은 이 유로는 적은 표본수로도 환자를 대상으로 하여 연구의 당위성이 확 보되기 때문이라고 사료된다. 그러나 적은 대상자로 계획한 연구는

Table 4. Methodological quality of trials

\begin{tabular}{|c|c|c|c|c|c|c|c|c|c|c|c|c|c|c|c|}
\hline & 연구 분류 & 저자 & Method & 1 & 2 & 3 & 4 & 5 & 6 & 7 & 8 & 9 & 10 & 11 & Score \\
\hline 1 & Neurological & Kim et al. (2017) & Clinical trial & Y & Y & Y & Y & Y & Y & Y & Y & Y & Y & Y & 10 \\
\hline 2 & Muscular skeletal & Lee and Kim (2019) & Clinical trial & Y & Y & $N$ & $\mathrm{~N}$ & N & N & N & Y & Y & Y & Y & 5 \\
\hline 3 & Muscular skeletal & Chi and Kim (2019) & Clinical trial & Y & Y & N & Y & N & N & N & Y & Y & Y & Y & 6 \\
\hline 4 & Muscular skeletal & Jeon and Kim (2017) & Clinical trial & Y & Y & Y & Y & Y & N & $\mathrm{N}$ & Y & Y & Y & Y & 8 \\
\hline 5 & Muscular skeletal & Lee et al. (2018) & Clinical trial & Y & Y & $N$ & Y & N & N & $\mathrm{N}$ & Y & Y & Y & Y & 6 \\
\hline 6 & Muscular skeletal & Kang and Kim (2019) & Clinical trial & Y & Y & $N$ & Y & $N$ & N & $\mathrm{N}$ & Y & Y & Y & Y & 6 \\
\hline 7 & Neurological & Ji et al. (2016) & Clinical trial & Y & Y & $\mathrm{N}$ & Y & $N$ & N & $\mathrm{N}$ & Y & Y & Y & Y & 6 \\
\hline 8 & Neurological & An and Park (2017) & Clinical trial & Y & Y & Y & $\mathrm{N}$ & $\mathrm{N}$ & N & Y & $N$ & Y & Y & Y & 6 \\
\hline 9 & Muscular skeletal & Jin et al. (2017) & Clinical trial & Y & Y & $\mathrm{N}$ & Y & $\mathrm{N}$ & N & $\mathrm{N}$ & Y & Y & Y & Y & 6 \\
\hline 10 & Muscular skeletal & Park and Kim (2018) & Clinical trial & Y & Y & $\mathrm{N}$ & N & $\mathrm{N}$ & N & $\mathrm{N}$ & Y & Y & Y & Y & 5 \\
\hline 11 & Muscular skeletal & Jang et al. (2019) & Clinical trial & Y & Y & $\mathrm{N}$ & $\mathrm{N}$ & $\mathrm{N}$ & N & $\mathrm{N}$ & Y & Y & Y & $\mathrm{Y}$ & 5 \\
\hline 12 & Neurological & Choi and Kim (2019) & Clinical trial & Y & Y & $\mathrm{N}$ & Y & N & N & $\mathrm{N}$ & $\mathrm{N}$ & Y & Y & Y & 5 \\
\hline 13 & Muscular skeletal & Kim and Kim (2019) & Clinical trial & Y & Y & Y & Y & Y & Y & $\mathrm{N}$ & Y & Y & Y & Y & 9 \\
\hline 14 & Muscular skeletal & Choi et al. (2017) & Clinical trial & Y & Y & $\mathrm{N}$ & Y & $\mathrm{N}$ & N & $\mathrm{N}$ & Y & Y & Y & Y & 7 \\
\hline 15 & Neurological & Lee et al. (2019) & Clinical trial & Y & Y & $N$ & Y & N & N & N & Y & Y & Y & Y & 6 \\
\hline 16 & Neurological & Kim and Kang (2019) & Clinical trial & Y & Y & $\mathrm{N}$ & Y & N & N & $\mathrm{N}$ & Y & Y & $\mathrm{Y}$ & $\mathrm{Y}$ & 6 \\
\hline
\end{tabular}

PEDro items: 1 eligibility criteria, 2 random allocation, 3 concealed allocation, 4 baseline comparability, 5 blind subjects, 6 blind therapist, 7 blind assessor, 8 adequate follow-up, 9 intention to treat analysis, 10 between-group statistical comparisons, 11 point estimates and variability. 
임상적으로 유의한 차이를 얻지 못할 수도 있기 때문에 이 또한 지양 해야 하며, 연구의 신뢰성을 높이기 위해선 표본수의 명확함을 필요 로 한다.

G-power는 효과크기를 선정해 표본수를 제공하여 실험연구에서 많이 사용되고 있으며 본 연구에서도 69편의 논문 중 62 편 $(90 \%)$ 이 실 험연구로 나타났다. 많은 논문들이 실험연구로 분류됨에 따라 연구 수준을 분석한 결과 제 3 수준 $(58 \%)$, 제 2 수준 $(32 \%)$, 제 4 수준 $(6 \%)$, 제6 수준(4\%) 순으로 나타났다. 이러한 결과로 보았을 때 G-power를 사용 한 연구는 제 2,3 수준에 많이 분포하며, 높은 수준의 연구라고 할 수 있다. 그러나 비교적 표본수가 적거나, 즉시적인 효과를 측정한 것으 로 나타난 제 4,6 수준의 연구는 결과를 일반화시키는데 제한이 따르 기 때문에 낮은 수준의 연구로 평가된다. 가장 높은 단계인 체계적 고 찰이나 메타분석연구에 해당하는 제 1 수준의 연구는 없었다. 체계적 고찰과 메타분석연구는 적절한 문헌을 검색하고, 연구물의 연구설 계와 특성을 조사하는 연구이다. ${ }^{36}$ 본 연구에서 선정한 3 곳의 등재지 에서도 3 년간 788 편의 많은 논문이 출판되었다. 이렇게 양적이나 방 법론적인 면에서 연구들의 많은 발전이 이뤄져 왔기 때문에 추후에 제1수준에 해당하는 연구가 중요영역으로 자리 잡아 더 많아질 것으 로 생각된다. 최근 10 년 이내에 Zorcolo 등 37 , Warth 등 ${ }^{38}$, Du 등 ${ }^{39}$ 과 같 이 G-power를 사용한 제1수준의 연구들이 나오고 있으며, Lee 등 ${ }^{40}$ 의 연구로 보아 국내 연구자들 또한 G-power를 사용해 제 1 수준의 연구 를 진행함을 확인할 수 있었다. 이로 보아 향후 G-power를 사용한 높 은 수준의 연구들이 계속해서 늘어날 것으로 예상된다.

본 연구에서는 연구의 질을 평가하기 위해 PEDro scale을 사용하였 으며, 선정기준에 따라 16 편의 논문이 평가 되었다. 16 편의 논문 중 신 경계 논문은 6 편, 근골격계 논문은 10 편이었다. 신경계 논문 중 성인 을 대상으로 한 논문 5 편은 균형 및 보행에 미치는 영향을 보는 연구 가 대부분이었으며 그 외에 평가된 항목으로는 낙상위험도, 체간 조 절, 심리사회적 수준에 미치는 영향이 있었다. 뇌성마비 아동을 대상 으로 한 연구는 진동자극 훈련이 근두께의 변화와 대동작 운동기능 에 미치는 영향을 보는 연구였다. 근골격계 논문에서는 목과 어깨의 통증과 가동범위를 본 연구가 6 편으로 가장 많았다. 통증과 가동범 위 외에 평가 된 항목으로는 근력, 자세, 기능장애 지수를 평가하였으 며 중재방법은 테이핑 적용, 감각 운동, 관절가동술 등으로 다양했다. 나머지 4편의 연구는 호흡재활 프로그램이 가로막 움직임과 호흡기 능에 미치는 영향, 허리의 통증과 골반의 움직임 및 균형에 대한 스트 레칭 및 강화운동의 효과, 무릎 인공관절 수술을 한 환자를 대상으 로 가상현실 기반의 게임훈련을 적용했을 때 통증과 가동범위 및 균 형에 미치는 영향, 전신진동자극 훈련이 발목의 불안정성과 가동범 위 및 균형에 미치는 영향을 조사한 연구였다. ${ }^{20-35} 16$ 편 논문의 PEDro scale의 평균점수는 6.37 이었다. 구체적으로는 매우 좋음에 해당하는
논문 2 편, 좋음에 해당하는 논문 10 편, 보통은 4 편이었다. 선행연구에 서 Keyword로 뇌졸중을 검색하여 나온 논문 중 무작위 대조군 실험 272편을 선정하여 PEDro scale을 적용하였을 때, 그 평균점수는 5.78 점으로 나타났다. ${ }^{41}$ 본 연구에서 평가한 16 편 논문의 평균점수는 6.37 점으로 좋음에 속하며, 앞의 연구 272 편에 비해 논문 수는 적지만, 평 균점수를 비교해 보면 높은 편에 속한다. 이는 G-power를 사용해 표 본수를 선정하여 진행 된 연구에서 연구의 질이 전반적으로 높았던 것으로 판단된다. 대부분의 임상실험에서 물리치료 중재를 할 때 치 료사와 대상자에게 눈가림법(blind)과 같은 엄격한 실험방법을 적용 하기는 어려운 실정이며, 이는 PEDro scale 적용시 낮은 점수를 받게 된다.42 본 연구에서 평가한 논문들 역시 대부분의 연구가 대상자, 치 료사, 평가자에 대한 눈가림법이 시행되지 않았고, 그에 따라 낮은 점 수가 부여되어 좋음과 보통 수준의 연구로 분류되었다.

낮은 검정력을 가진 연구는 중요한 결과를 얻지 못하고 임상 관련 효과를 탐지하지 못하기 때문에 표본수 계산은 적절한 검정력을 제 공하는데 필수적이다..$^{15}$ 본 연구에서 연구의 질 평가를 위해 선정된 논문은 환자를 대상으로 한 연구와 RCT논문이었고, PEDro scale 평 가를 위해 배제된 논문은 건강한 성인을 대상으로 한 연구와 질환이 없는 노인을 대상으로 한 연구였다. PEDro scale을 사용해 분석한 이 전 연구를 보았을 때, 근골격계의 비율이 높았으며 그 다음 심폐계, 신경계 비율로 나타났다. ${ }^{43}$ 본 연구에서도 근골격계 10 편, 신경계 6편 으로 근골격계 논문이 많았다. 그 중 신경계 논문은 눈가림법을 시행 한 논문이 절반도 안되었는데, 실험연구에서 눈가림법을 적용하는 것은 엄격한 실험설계를 필요로 하기 때문이라고 생각된다. 본 연구 의 제한점은 다음과 같다. 최근 5 년의 연구를 선정하여 분석하였지 만 국내의 모든 학회지에 투고된 연구를 분석하지는 못했다. 또한 연 구형태의 분류가 다양하지 않았기 때문에 특정 연구형태에서 Gpower를 사용한 연구의 동향 분석이 어려웠다. 향후 다양한 연구형태 에서 G-power를 사용한 연구 동향 파악에 관한 추가적인 연구가 진 행되어 G-power 사용이 각 연구형태에서 효과적인 표본수를 제공한 다는 근거를 마련할 필요가 있다고 사료된다.

높은 근거수준의 연구를 위해서는 최대한 적절한 대상자 확보를 해야 한다. 그러나 연구자들이 대상자 확보를 위해 표본수 크기를 늘 릴 수 있는 여력이 없을 수도 있으며, 연구자들은 대상자 수를 늘렸을 때 발생하는 경제적 손해도 고려해야한다. G-power는 효과적인 표본 수를 얻을 수 있게 하여 신뢰성 있는 검출이 가능하도록 도와준다. ${ }^{44}$ 본 연구의 결과와 같이 G-power를 사용한 연구에서는 높은 수준의 연구가 많았고, 연구의 질 또한 좋음에 속해 있었다. 따라서 G-power 를 사용해 얻은 효과적인 표본수는 연구자가 더욱더 높은 근거수준 의 연구를 할 수 있게 하는 방법 중 하나라고 생각된다. 


\section{REFERENCES}

1. Kim SJ, Kim YS, Kim JH et al. A study on the development of convergence education program for workers in health and medical industry in preparation for the forth industrial revolution. Journal of the Korea Convergence Society. 2018;9(5):43-52.

2. Maeng JY. A Study on Alternatives to Link and Converge Robot Industry and Cultural Arts Industry. The Catholic Universicy of Korea. Dissertation of Master Degree. 2017.

3. Hyun JW. A Study on the Development of ROK Military in the Fourth Industrial Revolution. Daejin University Graduate School. Dissertation of Master Degree. 2019.

4. Lee D. Design of A Clinically Relevant Elbow Exoskeleton Robot for Stroke Patients. Department of Robotics Engineering. Dissertation of Master Degree. 2015.

5. Lee HK. The Effects of Robot-Assisted Gait Training with Body Weight Support on Gait and Balance in Stroke Patients. Gachon University. Dissertation of Master Degree. 2016

6. Goo BO, Lee SY, Kim KH et al. The necessity of independent clinic open by physical therapist in South Korea. J Korean Soc Phys Med. 2010;5(2): 143-50.

7. Choi YI, Park EY. A methodological quality assessment of meta-analyses of rehabilitation therapy areas in Korea. Asia-pacific Journal of Multimedia Services Convergent with Art, Humanities, and Sociology. 2014;4(2):71-8.

8. Kim K, Park ES, Cho YH et al. A study on the development of standard curriculum for physical therapy in Korea. J Korean Soc Phys Ther. 2006;18(6):23-32.

9. Im TB. Qualitative methodology: approach and application. Journal of Governmental Studies. 2009;15(1):155-88.

10. Yeon HS, Mo NJ, Min MS et al. A comparison of sample size requirements for intraclass correlation coecient (ICC). Yonsei University Collegeof Medicine. 2010;23(3):497-510.

11. Moher D, Dulberg CS, Wells GA. Statistical power, sample size, and their reporting in randomized controlled trials. JAMA. 1994; 272(2):122-4.

12. Freiman JA, Chalmers TC, Smith H Jr et al. The importance of beta, the type II error and sample size in the design and interpretation of the randomized control trial. Survey of 71 "negative" trials. N Engl J Med. 1978; 299(13):690-4.

13. Guo Y, Logan HL, Glueck DH et al. Selecting a sample size for studies with repeated measures. BMC Med Res Methodol. 2013;13:100.

14. Kang H. Sample size determination for repeated measures design using G Power software. Anesth Pain Med. 2015;10:6-15.

15. Abdul Latif L, Daud Amadera J. E, Pimentel D et al. Sample size calculation in physical medicine and rehabilitation: a systematic review of reporting, characteristics, and results in randomized controlled trials. Arch Phys Med Rehabil. 2011;92(2):306-15.

16. Case-Smith J PC. Research literature in occupational therapy, 20012005. Am J Occup Ther. 2008;62(4):480-6.

17. Arbesman M SJ, Lieberman D. Using AOTA's critically appraised topic (CAT) and critically appraised pater (CAP) series to link evidence to practice. OT Practice 2008;13(5):18-22.

18. Sackett DL RW, Gray JA et al. Evidence based medicine: what it is and hat it isn’t. Br Med J. 1996;312(7023):71-2.
19. Maher CG, Sherrington C, Herbert RD et al. Reliability of the PEDro scale for rating quality of randomized controlled trials. Phys Ther. 2003; 83(8):713-21.

20. Kim CY, Kim HD. Comparison of sensorimotor training using chintuck exercise with therapeutic stretching training on neck pain and mobility in individuals with chronic Non-Specific Neck Pain: a pilot randomized controlled trial. J Korean Soc Phys Med. 2019;14(2):29-40.

21. Lee HB, Choi HS, Shin WS. Effect of game-based balance training with CIMT on pain, muscle strength, range of motion, and dynamic balance in female patients with total knee replacement. J Kor Phys Ther. 2018; 30(5):159-65.

22. Park SJ, Kim SY. The effect of scapular dynamic taping on pain, disability, upper body posture, and range of motion in the postoperative shoulder. J Korean Soc Phys Med. 2018;13(4):149-62.

23. Jang MS, Choung SD, Shim JH et al. Effect of virtual reality inspiratory muscle training on diaphragm movement and respiratory function in female patients with thoracic restriction. J Korean Soc Phys Med. 2019;14(1):101-10.

24. Kim YG, Kang SH. The effect of virtual reality-based exercise program on balance, gait, and falls efficacy in patients with parkinson's disease. J Korean Soc Phys Med. 2019;14(4):103-13.

25. Jin YS, Choi YH, Shim JK et al. Effect of wholebody vibration stimulation according to various frequencies on ankle instability, ankle range of motion and balance ability in adult with chronic ankle instability. J Korean Soc Phys Med. 2018;13(1):63-72.

26. Jeon HC, Kim GW. Effectiveness of global postural reeducation compared to segmental stretching on pain, disability, and QOL of subjects with neck and shoulder pain. J Kor Phys Ther. 2017;29(1):7-15.

27. Ji SG, Shin YJ, Kim MK. The effects of repetitive transcranial magnetic stimulation on balance ability in acute stroke patients. J Korean Soc Phys Med. 2016;11(3):11-7.

28. Chi CY, Kim SY. Effects of sling exercise with vibration on range of motion, muscle strength, pain, disability in patients with shoulder injuries. Phys Ther Korea. 2019;26(3):11-22.

29. Kang TW, Kim BR. The effects of stretching and strengthening exercise on the pain, pelvic tilt, functional disability index, and balance ability of patients with chronic lower back pain. J Kor Phys Ther. 2019;31(1): 7-12.

30. Lee HJ, Kim SY. Effects of thoracic mobility exercise on cervicothoracic function, posture and pain in individuals with mechanical neck pain. Phys Ther Korea. 2019;26(3):42-56.

31. An SH, Park DS. The effects of trunk exercise on mobility, balance and trunk control of stroke patients. J Korean Soc Phys Med. 2017;12(1):2533.

32. Choi SA, Cynn HS, Shin AR et al. Effects of verbal cue for scapular depression during scapular posterior tilt exercise on scapular muscle activities and clavicular tilt angle in subjects with rounded shoulder posture and upper trapezius myofascial pain. Phys Ther Korea. 2017; 24(3):30-9.

33. Lee WB, Lee HS, Park SW et al. Effects of whole body vibration training on lower limb muscle thickness and gross motor function in children with spastic cerebral palsy. J Korean Soc Phys Med. 2019; 14(4):195-201.

34. Choi GY, Kim SY. Impact of a fall-prevention exercise program on balance, gait, lower limb strength, and psychosocial characteristics in older adults with dementia. J Korean Soc Phys Med. 2019;14(1):75-89.

35. Kim SK, Kang TW, Park DH et al. Four-week comparative effects of ab- 
dominal drawing-in and diaphragmatic breathing maneuvers on abdominal muscle thickness, trunk control, and balance in patients with chronic stroke. Phys Ther Korea. 2017;24(3):10-20.

36. WJ S. An introduction of the systematic review and meta-analysis. Hanyang Med Rev. 2015;35(1):9-17.

37. Zorcolo L, Rosman AS, Restivo A et al. Complete pathologic response after combined modality treatment for rectal cancer and long-term survival: a meta-analysis. Ann Surg Oncol. 2012;19(9):2822-32.

38. Warth RJ, Dornan GJ, James EW et al. Clinical and structural outcomes after arthroscopic repair of full thickness rotator cuff tears with and without platelet-rich product supplementation: a meta-analysis and meta-regression. Arthroscopy. 2015;31(2):306-20.

39. Du J, Zhang W, Guo L et al. Two FSHR variants, haplotypes and metaanalysis in Chinese women with premature ovarian failure and polycystic ovary syndrome. Mol Genet Metab. 2010;100(3):292-5.
40. Lee YH, Bae SC, Song GG. The association between the functional PTPN22 1858 C/T and MIF - 173 C/G polymorphisms and juvenile idiopathic arthritis: a meta-analysis. Inflamm Res. 2012;61(5):411-5.

41. Bhogal SK, Teasell RW, Foley NC et al. The PEDro scale provides a more comprehensive measure of methodological quality than the Jadad scale in stroke rehabilitation literature. J Clin Epidemiol. 2005;58(7):668-73.

42. De Morton NA. The PEDro scale is a valid measure of the methodological quality of clinical trials: a demographic study. Aust J Physiother. 2009;55(2):129-33.

43. Elkins MR, Moseley AM. Societa italiana de fisioterapia and the physiotherapy evidence database (PEDro). Arch Physiother. 2019;9:5.

44. Perugini M, Gallucci M, Costantini G. A practical primer to power analysis for simple experimental designs. International Review of Social Psychology. 2018;31(1):20. 